\section{Understanding of the developing child}

Learning and the Development of Cognition. By Barbel Inhelder, Hermine Sinclair and Magali Bovet. Translated by Susan Wedgwood. Pp. xiv +308 . (Routledge and Kegan Paul: London, February 1975.) $£ 4.95$.

Piaget's theory is frequently presented as a rigid maturational system in which stage follows stage, more or less regardless of the child's social milieu, so that specific training procedures to speed up intellectual development are ineffective and pointless: either the child has the developmental structures to assimilate the material, in which case nothing substantially new can be acquired, or else those structures are not yet developed and no useful learning can take place.

No wonder that psychologists and educators alike, in their efforts to understand and to instruct the developing child, recoil from this altogether pessimistic picture and put it aside as irrelevant. No wonder, either, that any experimental evidence of successful intervention programmes in teaching mental operation at a younger age than commonly expected is flaunted as proving Piaget's theory wrong. Fortunately, the interpretation I have mentioned is but a caricature of the theory, born out of the unquestioned assumption that between a hereditarily given, unchanging potential and learning in the form of storing and coding environmental information there are no other factors contributing to the growth of intelligence. Piaget rejects both extremes of the heredity-environment controversy without lapsing into an interactionist stance in which one is locked in interminable arguments about the correct statistical proportions, but by proposing mental development as a psychological process altogether different from the learning of information or the unfolding of instinctual patterns.

This book, if nothing else, is a reminder that Piaget's theory is not at all aversive to a study of environmental input as it affects children's efforts at understanding their world. On the contrary, the investigations reported are predicated on the idea that developmentally suitable encounters will bring about intellectual progression; yet there is no concomitant desire of speeding up, rather of observing the developmental process in a concrete setting. The authors, who are Piaget's closest collaborators of many years, conceptualise this progression as a construction on the part of the child in the face of fluctuations and conflicts between apparently divergent schemes of thinking. The encounters are devised precisely to expose children, if possible, to an awareness of this conflict and thus ti bring about a restructuring of these schemes; in other words, a progression from a base of less or conflicting understanding to a stage at which the conflicting views are subsumed as nondisturbing facets of a more adequate understanding. As an illustration, in the comparison of a W-shaped line and a straight line, the length of the lines and the number and configuration of segmental units making up the lines can create a conflict in a child who does not fully coordinate the concept of length in relation to segmentation and unit element.

In all experiments the children were carefully selected as being in a transitional phase and tested before and after training; each child's performance was individually analysed and tentative categories of progress-or lack of itwere proposed. Though the methods of experimentation include the use of such familiar concepts as assessment of the quantity of a liquid, numbers, inclusion, conservation of substance and weight, the thinking activities to which the children were exposed are ingenious and original, not only in helping the child's progress, but also in helping us to understand and observe the process of development. In the foreword Piaget singles out some of the more important theoretical insights to be gleaned from this research which is frankly acknowledged to be but an exploratory beginning in the search towards the unravelling of the process of development.

Thus the book is another milestone in Genevan research and contains the clearest exposition so far of Piaget's elusive concept of developmental equilibration. One may add to this that the translation was supervised by one of the authors and is not full of mistranslations as are, unfortunately, many other works on Piaget's theory. But make no mistake, like other Genevan writings it is not easy reading and it does have weaknesses for which the Genevans can be so easily criticised: points of empirical and methodological inadequacy, incomplete protocols, theoretical overinterpretations. A reader who cannot see beyond these will no doubt be dissatisfied. But those who want to understand better the nature of environmental occasions leading to intellectual development, and who search for guidelines in worthwhile psychological research will find here an exciting theoretical framework, all the more so on account of its obvious practical and educational applications.

H. Furth

\section{Introduction to Marine Geology and Geomorphology CUCHLAINE A. M. KING}

During the ten years since the first edition of Oceanography for Geographers (distributed in the U.S.A. as "Introduction to Oceanography") was published, there has been an explosive development in nearly all aspects of the subject. So much has been written concerning this aspect of oceanography that it has been necessary for the author to prepare the new edition of Oceanography for Geographers as two separate books, both of which are almost entirely new. This is the first. Cloth $\mathbf{f} 9.90$ Paper

$£ 4.90$

\section{Introduction to \\ Physical and Biological Oceanography} CUCHLAINE A. M. KING

The physical aspects considered in this book include an introduction to the character of ocean water and its circulation in the form of surface currents and deep wave movements. The subjects of tides and the various waves that disturb the surface of the ocean are discussed and the biological aspects of oceanography form a major and topical theme through the second half of the volume.

$$
\text { Cloth } £ 11.00 \text { Paper } £ 5.50
$$

\section{Rural Recreation in the Industrial World}

\section{G. SIMMONS}

The provision of rural recreational facilities in industrialized societies is of growing economic and social significance. This book deals with the demand for outdoor recreation in industrial nations in the northern hemisphere during the last twenty years and the response to it in the development of facilities by public and private enterprise.

\section{The Ecology of Natural Resources \\ I. G. SIMMONS}

... good books require no apologies whatever for their subject .... why is his book so intellectually satisfying when others merely depress? One factor is the sense of order so crucial in a textbook... Far from making this a dull academic tome, it brings welcome relief from the ultimately more tedious Ehrlich-like stridency of many of Simmon's predecessors.' New Society

Cloth $\mathbf{8 . 0 0}$ Paper $£ 3.85$

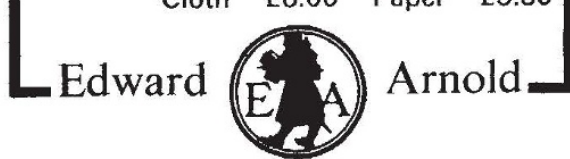

25 Hill Street, London W1X 8LL 